\title{
Fault effect analysis based on elements loadability to evaluate reliability in power systems
}

\author{
Mantilla Florez, B.H., Silva Ortega, J.I., Candelo Becerra, J.E.
}

\begin{abstract}
:
This paper presents a fault effect analysis (FEA) based on element loadability as a complement tool to evaluate reliability and identify vulnerable points in power systems. The steps of the method are the calculation of overloaded elements after contingencies, determination of the FEA based on element loadability, selection of critical overloaded elements, and evaluation of system reliability by considering the energy not supplied (ENS). A simplified transmission and distribution ( $T$ and $\mathrm{D}$ ) power system with 30 nodes was used as study case for the method. The results show that the loadability percentage of transformers and power lines are calculated successfully for the N-1 contingency analysis performed in the network and the method selects the elements with higher loadability values, evaluating the impact that each critical contingency generates on the power system reliability. The method can be used to prioritize remedial actions for critical elements on power systems.
\end{abstract}

Keywords: Power system reliability, Reliability, Silicon compounds, IEEE transactions, Silicon, Tools 